\title{
Investigations of calcium carbonate mineral precipitation in the presence of oil using 3D-printed micromodels
}

\author{
Menke, H.P. ${ }^{1}$, Patsoukis-Dimou, A. ${ }^{1}$, Maes, $\mathrm{J}^{1}{ }^{1}$, \\ McGravie, T. ${ }^{1}$, Singleton, M. ${ }^{1}$, MacKay, E. ${ }^{1}$, \\ GeIGER, S. ${ }^{1}$ \\ ${ }^{1}$ Institute for GeoEnergy Engineering, Heriot-Watt \\ University, Edinburgh, UK
}

Carbon storage in deep aquifers relies on permanent mineralisation of carbon dioxide as solid carbonate mineral. As injected $\mathrm{CO}_{2}$ leaves the well and travels further into the aquifer it mixes with the reservoir brine and initially becomes a low $\mathrm{pH}$ acid. However, as mixing and dilution continues, the $\mathrm{pH}$ increases, and the carbonate species become supersaturated and fall out of solution. This precipitation then fills the pore space of the reservoir with permanently stored carbonate mineral. The $\mathrm{CO}_{2}$ injection strategy must be designed so that precipitation is favourable at long time scales far away from the injection well, but not so fast that the major flow pathways through the aquifer are clogged and expensive additional well-drilling is required.

The interplay between mixing, oil saturation, particle nucleation and transport, and crystal growth processes are poorly understood. Here we use the combination of 3Dprinted micromodels, light microscopy, and pore-scale multiphase reactive transport models to investigate flow and transport conditions favourable for calcium carbonate precipitation. 3D-printed micromodels have the benefit of being inexpensive to produce with high design fidelity. In these experiments we inject mineral oil into 3D-printed micromodels filled with $\mathrm{CaCl}_{2}$ brine. We then follow drainage with imbibition using $\mathrm{NaHCO}_{3}$ brine that is chemically incompatible with $\mathrm{CaCl}_{2}$ while monitoring porescale displacements and precipitation using a visible light camera.

We see that the local saturation index and the oil-brine interface play a key role in both the location and movement of the precipitated particles and oil ganglia. We observe initially fast then slower imbibition as more pathways are cleared of oil. Areas with precipitated particles impede ganglia movement with thinning of oil layers and pinning of oil ganglia by precipitation. We also see dynamic rearrangement of oil clusters that result in additional mixing and precipitation with some pores becoming blocked with precipitated particles. These experimental results are then benchmarked with direct numerical simulations using GeoChemFOAM. This work has extensive applications for the carbon storage and enhanced oil recovery industries. 